\title{
Ocular Defect: A Prosthodontic Challenge
}

\author{
Poonam Malik ${ }^{1, *}$, Manu Rathee ${ }^{1}$, Jyotirmay Singh ${ }^{2}$ \\ ${ }^{1}$ Department of Prosthodontics, Post Graduate Institute of Dental Sciences, Pt. B.D.Sharma University of Health Sciences, Rohtak, \\ Haryana \\ ${ }^{2}$ Department of Orthodontics, Dr.B.R.Ambedkar Institute Of Dental Sciences and Hospital, Patna \\ *Corresponding author: drpoonammalik82@yahoo.co.in
}

Received September 26, 2014; Revised October 05, 2014; Accepted October 20, 2014

\begin{abstract}
The disfigurement associated with the loss of an eye can cause significant physical and emotional problems. This article describes the rehabilitation of an orbital defect with a silicone prosthesis, wherein retention has been achieved by a combination of anatomic undercut, adhesives and eyeglasses.
\end{abstract}

Keywords: eye prosthesis, ocular defect, ocular impression

Cite This Article: Poonam Malik, Manu Rathee, and Jyotirmay Singh, "Ocular Defect: A Prosthodontic Challenge.” International Journal of Dental Sciences and Research, vol. 2, no. 5 (2014): 120-122. doi: 10.12691/ijdsr2-5-5.

\section{Introduction}

The unfortunate absence or loss of an eye may be caused by a congenital defect, irreparable trauma, a painful blind eye, sympathetic ophthalmia, or the need for histologic confirmation of a suspected diagnosis [1]. Two surgical procedures are generally involved, one is evisceration which consists of removal of the contents of the globe, but leaving the sclera and on occasion the cornea in place and the other is enucleation where the eyeball itself is removed [2].

Replacement of the lost eye as soon as possible after healing from eye removal is necessary to promote physical and psychological healing for the patient and to improve social acceptance. A multidisciplinary management and team approach are essential in providing accurate and effective rehabilitation and follow up care for the patient [1]. This article describes a simplified method for fabrication of a silicone orbital prosthesis.

\section{Case Presentation}

A 35-year-old male reported with the chief complaint of missing right eye. The patient gave history of enucleation of the eye due to accidental exposure to nitric acid. On examination a bare fibrosed ocular socket covered by grafted skin was seen. (Figure 1) Also, the patient did not report of any pain or discomfort of the periorbital tissue. A treatment was planned for fabricaton of a custom silicone orbital prosthesis.

\section{Clinical Technique}

1. A facial impression was made using irreversible hydrocolloid with plaster of Paris reinforcement. (Figure 2) Following this a cast was poured in dental stone.

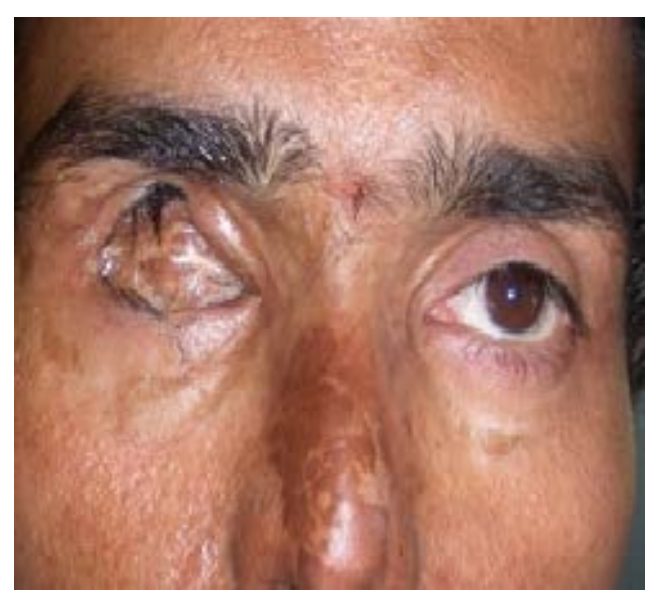

Figure 1. Pre-operative view

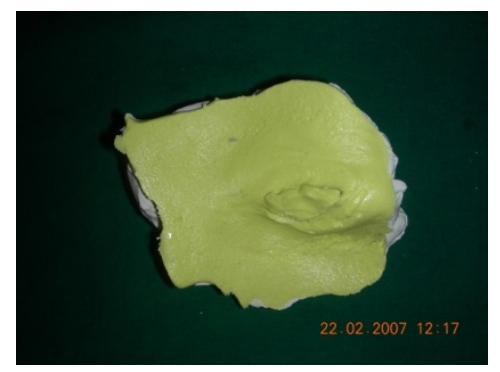

Figure 2. Ocular Impression

2. Then a suitable stock ocular prosthesis was selected that closely approximates the colour, size and shape of the iris and sclera of the other eye. The stock ocular prosthesis was placed on the cast and the periphery was arbitrarily trimmed to match socket border extensions on the cast.

3. Next, the ocular prosthesis was positioned to simulate the positioning of the left eye, with the patient focusing on the distant point directly ahead. A reference mark was place at the midline and a Boley's gauge was used to verify the mediolateral placement. The pupils were 
used as reference points for evaluation. Correct mediolateral, anteroposterior and inferosuperior positioning of the prosthesis was done to exactly mimic the position of the normal eye.

4. Some amount of baseplate was then added on the tissue side of the prosthesis so as to orient the prosthesis to the desired visual axis.

5. Then a thin even layer of tissue conditioner (Soft liner; GC Corporation, Tokyo, Japan) was applied on the tissue surface of the prosthesis and placed back into the socket. The prosthesis was left in situ for 20 minutes during which the patient was asked to perform various functional movements.

6. The modified prosthesis was then invested and packed with tooth colored heat cure acrylic resin. (Figure 3) Slow curing was carried out for acrylisation at $74^{\circ} \mathrm{C}$ $\left(165^{\circ} \mathrm{F}\right)$ for 8 hours.

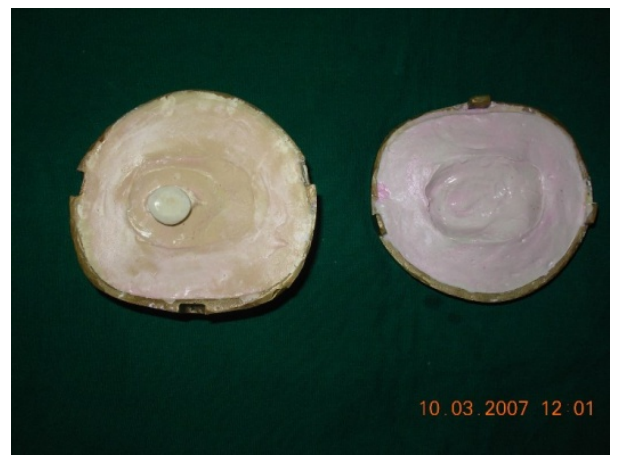

Figure 3. Processing of ocular prosthesis

7. After curing the ocular prosthesis was recovered and polished. At the next appointment the modified stock ocular prosthesis was inserted and evaluated again for patient comfort and esthetics.

8. Then sculpting of the periorbital tissues was effected. The lid contours and periorbital tissues were mimicked to those of the left eye as closely as possible. The lines of juncture were feathered.

9. The appropriate colours were then obtained by mixing different shades with the silicone material, following which it was packed and then processed.

10. The prosthesis was retrieved, trimmed and adjusted according to the needs of the patient. Prosthetic eyelashes were then attached to the upper lid. Since the lower eyelashes are quite scanty, their presence was simulated with a few vertical lines of extrinsic painting on the lower lid.

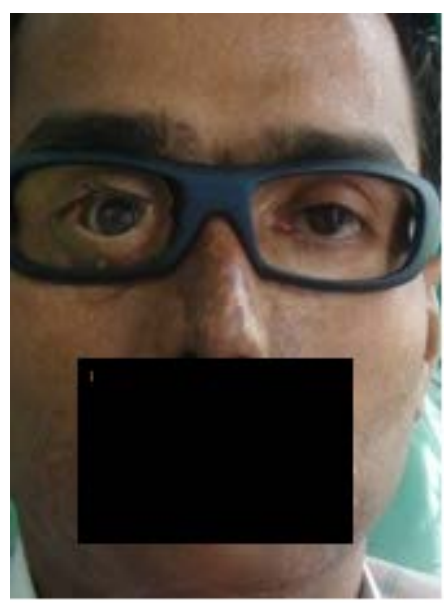

Figure 4. Post-operative view
11. The retention was achieved with the help of skin adhesive and by engagement of orbital undercut with the flexible material. Additional retention was provided by means of eyeglass frame attached to the prosthesis (Figure 4).

\section{Discussion}

The importance of an orbital prosthesis with acceptable esthetics and reasonable motility in restoring normal appearance in patients with anophthalmia has long been recognized. [1] The need for an artificial eye can sometimes be satisfied by stock ocular prosthesis which is available in standard sizes, colours and shapes. These are relatively inexpensive and can be delivered quickly. Often, however, a custom ocular prosthesis is indicated. Advantages include better adaptation to underlying tissues, greater mobility of the prosthesis, better facial contours, and enhanced esthetics gained from control over the size and color of the pupil and iris. Nonetheless, a custom prosthesis is more expensive than a stock prosthesis, and several steps are required for its fabrication. [3] Therefore a modified stock ocular prosthesis is a good alternative which is relatively inexpensive and easy to fabricate.

Accurate alignment of the artificial eye is one of the major prerequisites for aesthetic success of orbital prosthesis. Facial measurements and various devices have been proposed for orienting the ocular portion of the orbital prosthesis [4,5].

Pare also used glass and porcelain for eyes, which was a great step forward. [5] Methyl methacrylate prosthesis became popular as they offered superior strength and could be modified in shape and size. Flexible material like silicone became advantageous when the defect extends beyond the orbital area and encounters movable tissue beds.

The rehabilitation of the orbital defect is a complex task, and if reconstruction by plastic surgery is not possible or not desired by the patient, the defect can be rehabilitated by an orbital prosthesis. Retention of the orbital prosthesis can be achieved using adhesives, attachments to eyeglasses, or engagement of hard or soft tissue undercuts [6].

The use of osseointgrated implants is a popular approach, as it offers improved retention compared to the existing alternatives. Various factors including systemic conditions and financial constraints happen to limit the use of osseointegrated implants in few patients [7].

\section{Conclusion}

This article describes the rehabilitation of an orbital defect with a silicone prosthesis, wherein retention has been achieved by a combination of anatomic undercut, adhesives and eyeglasses.

A simple technique for the fabrication of a silicone orbital prosthesis with the use of modified stock ocular prosthesis is described. It allows for proper positioning of the iris. Also, allows the finished orbital prosthesis to generate an equal distribution of pressure throughout the defect, while increasing its movement due to excellent adaptation to the walls of the socket.

\section{Ethics Statement}


The consent of the patient was sought prior to and approved the inclusion of his case and his photographs in this study.

\section{Conflict of Interest}

No conflict of interest was declared.

\section{References}

[1] Artopoulou I, Montgomery P, Wesly P, Lemon J; Digital imaging in the fabrication of ocular prosthesis. J Prosthet Dent 2006; 95: 327-30.
[2] Taylor T. Clinical Maxillofacial Prosthetics; 2000 Quintessence Publishing Co, Inc p. 233-76.

[3] Mathews M. F, Smith RM, Sutton A J, HudsonR: The ocular impression: A review of the literature and presentation of an alternate technique. J Prosthodont 2000; 9: 210-216.

[4] Chalian V A, Drane JB, Standish SM. Maxillofacial prosthetics. Multidisciplinary practice. Baltimore: Williams and Wilkins, 1971; 294-304.

[5] Gibson T. The prosthesis of Ambroise Pare. Br J Plast Surg 1955; 8: 3-8.

[6] Beumer J, Curtis TA, Firtill DN. Maxillofacial rehabilitation. Prosthodontic and surgical considerations. St Louis: C V Mosby; 1979; 364-71

[7] Pine, K.R., Sloan, B.H., Jacobs, R.. A proposed model of the response of the anophthalmic socket to prosthetic eye wear and its application to the management of mucoid discharge. Med. Hypotheses 2013; 81, 300-305. 